\title{
Fibrosis hepática congénita: un espectro clínico variable. Casos clínicos
}

\author{
Paul Harris $D^{1}$, Daniel Fodor $0^{a}$, Felipe Cavagnaro $S M^{2}$, \\ Marcia Di Egidio $\mathrm{S}^{1}$, Ignacio Duarte $\mathrm{G} \mathrm{De} \mathrm{C}^{3}$, Mario Fava $\mathrm{P}^{4}$. \\ Congenital hepatic fibrosis. \\ Report of five cases
}

Background: Congenital hepatic fibrosis (CHF) is an autosomic dominant disease that has been associated with polycystic kidney disease. Aim: To describe the medical management of 5 children with $\mathrm{CHF}$ and to evaluate the presence and extension of the associated renal disease. Patients and methods: Retrospective review of the medical charts of 5 children with CHF, aged 2 to 14 years. Results: Three children presented autosomic recessive polycystic kidney disease, which was diagnosed before the appearance of liver disease manifestations. They presented a more severe liver damage, with a more aggressive clinical course requiring use of transjugular intrahepatic porto-systemic shunts (TIPS) or surgical porto-systemic shunts to control portal hypertension. The other two children, in whom the diagnosed was based on asymptomatic hepatomegaly, had normal renal function and structure with a more benign clinical course. Conclusions: The diagnosis of CHF should be suspected not only in children with polycystic kidney disease but in those children with persistent, hard consistency, left lobe predominance hepatomegaly (Rev Méd Chile 2004; 132: 733-41).

(Key Words: Hypertension, portal; Liver cirrhosis; Polycystic kidney, antosomal dominant; Portasystemic shunt, surgical; Portasystemic shunt, transjugular intrahepatic)

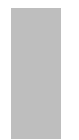

\begin{abstract}
Recibido el 22 de octubre, 2003. Aceptado en versión corregida el 15 de abril, 2004.
Departamento de Pediatría, Secciones de Gastroenterología ${ }^{1}$ y Nefrología ${ }^{2}$; Departamentos de Patología ${ }^{3}$ y Radiología ${ }^{4}$, Facultad de Medicina, Pontificia Universidad Católica de Chile. anterno de la carrera de medicina, Facultad de Medicina. PUC.
\end{abstract}

L a fibrosis hepática congénita (FHC) es una enfermedad hereditaria, autosómica recesiva, caracterizada por espacios porta fibróticos y alargados, que contienen múltiples conductos biliares dilatados, comunicados con el árbol biliar y cuya principal consecuencia es la hipertensión por-

Correspondencia a: Dr. Paul Harris D. Departamento de Pediatría, Facultad de Medicina, Pontificia Universidad Católica de Chile. Marcoleta 391, Santiago, Chile. C.P. 6510273. Tel: 3543340. Fax: 6331457.

E-mail: pharris@med.puc.cl $\operatorname{tal}^{1,2}$. En esta patología no sólo hay fibrosis, sino que la proliferación de los ductos biliares es un componente esencial de la lesión ${ }^{2,3}$. Clásicamente, la FHC ha sido considerada como una manifestación clínica tardía en la evolución de niños portadores de enfermedad renal poliquística autosómica recesiva (ARPKD, por su sigla en inglés), caracterizada por dilatación no obstructiva de los túbulos colectores. El mecanismo del desarrollo de múltiples ductos biliares es aún desconocido, habiéndose planteado la hipótesis de una disgenesia o desproporcionado crecimiento del epitelio 
biliar. También se ha sugerido que este mecanismo podría jugar un rol importante en el desarrollo de dilataciones en los túbulos colectores en el riñón ${ }^{3}$.

El objetivo de este trabajo es presentar una serie clínica con cinco niños que presentaron FHC, de los cuales dos no presentaron alteraciones ecográficas ni de función renal, poniendo de manifiesto la existencia de pacientes con las lesiones hepáticas características pero sin compromiso renal evidente. Además, se describe la utilización de la técnica de derivación portosistémica intrahepática transyugular (TIPS, por su sigla en inglés) en el manejo de la hipertensión portal (y sus complicaciones) en dos de estos niños.

\section{CASOS CĹNICOS}

Caso 1: Niña de 12 años, asintomática, que en un examen físico de rutina se le detectó hepatomegalia de $4 \mathrm{~cm}$ bajo el reborde costal, de consistencia normal. Se realizó un estudio bioquímico general que mostró una función hepática normal y una ecografía abdominal que confirmó hepatomegalia difusa, con alteración en la textura del parénquima hepático y signos iniciales de hipertensión portal. Se realizó una biopsia hepática percutánea que mostró distorsión parcial de la arquitectura por la presencia de septos fibrocolágenos, conteniendo numerosos conductos biliares irregulares revestidos por epitelio normotípico, muchos de ellos dilatados y rodeados por parénquima hepático normal (Figura 1). Una endoscopia digestiva alta mostró várices esofágicas grado II. En el proceso diagnóstico, se realizó una resonancia magnética, observándose el hígado aumentado de tamaño, siendo más evidente en el lóbulo izquierdo y caudado, de contornos moderadamente irregulares, con estructura interna homogénea, sin nódulos en el espesor del parénquima y sin dilatación de los conductos biliares intrahepáticos; el calibre de la vena porta y sus ramas principales estaba conservado, con venas suprahepáticas permeables, vesícula y vía biliar normal, bazo aumentado de tamaño, con presencia de colaterales esplenorrenales, además de presencia de várices esofágicas. Con el diagnóstico clínico e histológico confirmado de FHC, se reevaluó dirigidamente el tamaño y presencia de quistes renales (en la ecografía y resonancia magnética antes descritas) y función renal, encontrándose ambos normales. El cuadro clínico de la paciente ha sido manejado médicamente y las várices esofágicas no han sangrado, manteniéndose en profilaxis primaria con propanolol.

Caso 2: Niña de 2 años 6 meses, con hepatomegalia asintomática, pesquisada en un examen físico de rutina en un control pediátrico. El hígado se palpó a $2 \mathrm{~cm}$ bajo el reborde costal, pero de consistencia dura y el bazo se palpó a 3$4 \mathrm{~cm}$ bajo el reborde costal. El estudio inicial

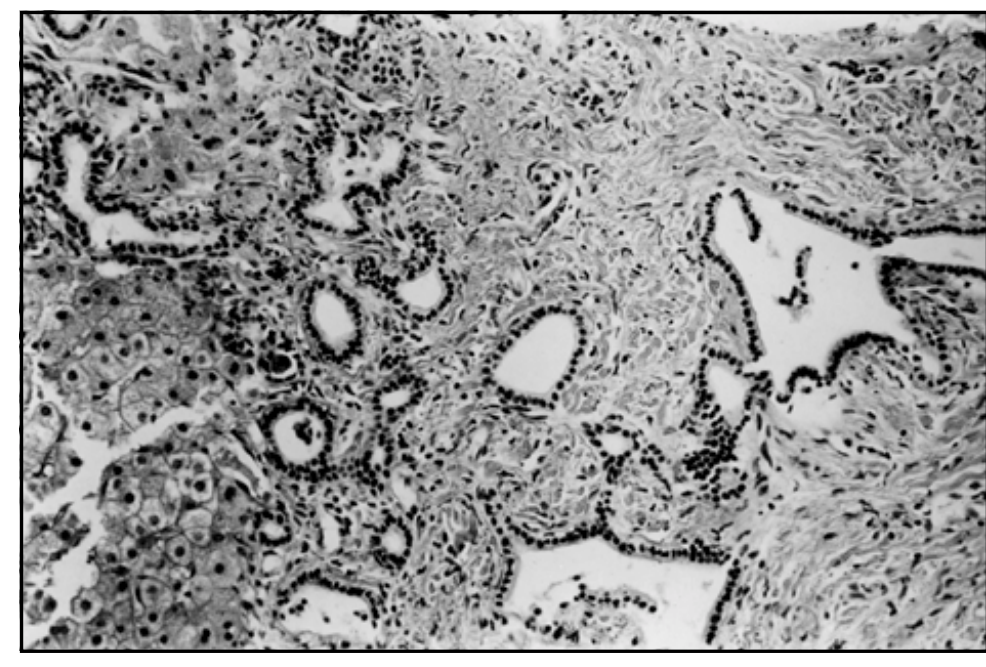

Figura 1. Biopsia hepática por aguja. Espacio porta ensanchado y fibroso, con conductos biliares múltiples, ramificados e irregulares, algunos con lumen amplio. En el ángulo inferior izquierdo, trabéculas hepáticas (Hematoxilina-eosina x 200). 
mostró un hemograma y un perfil bioquímico en rango normal. En el perfil lipídico destacaba colesterol de $205 \mathrm{mg} \%$ y triglicéridos de 192 $\mathrm{mg} \%$. El padre de la paciente estaba en tratamiento por dislipidemia. Debido a la persistencia de la hepatomegalia y sin un diagnóstico etiológico, se realizó biopsia hepática percutánea. El análisis histológico mostró espacios porta con aumento de tamaño y expandidos por acentuada fibrosis, con tendencia a circunscribir pequeños grupos de hepatocitos; también se observaron conductillos biliares de formas irregulares, angulosos, ramificados, algunos anastomosados y con proyecciones intraluminales del epitelio y estroma circundante (Figura 2). La niña no presentó compromiso renal, basado en pruebas de función renal y estudio de imágenes (ecografía renal). Una endoscopia digestiva alta mostró ausencia de várices esofágicas. Actualmente la paciente se encuentra asintomática y sin tratamiento profiláctico debido a la ausencia de várices esofágicas.

Caso 3: Niña de 8 años de edad, con antecedente de ARPKD, diagnosticada al año de edad, que evolucionó hacia la insuficiencia renal crónica terminal y requirió trasplante renal con donante vivo relacionado dos años más tarde, mientras permanecía en diálisis peritoneal crónica. A los 5 años fue referida a gastroenterología por hepatomegalia no dolorosa. El hígado se palpaba a $6 \mathrm{~cm}$ bajo el reborde costal, principalmente en el lado izquierdo; la función hepática era normal y no tenía signos de daño hepático. Se realizó una ecografía con Doppler hepático que mostró un hígado de tamaño límite normal, con crecimiento del lóbulo izquierdo, con vena porta y sus ramas pequeñas permeables y con flujo inicial hepatópeto, sin observarse quistes 0 alteraciones del parénquima. La evaluación endoscópica mostró várices esofágicas grado II a III iniciándose profilaxis con propanolol. Con el fin de definir el diagnóstico, se realizó una biopsia hepática percutánea que mostró hallazgos compatibles con FHC y descartó una enfermedad de Caroli, dada la presencia de amplios espacios porta septales, ensanchados, fibrosos, ramificados y con conductos biliares irregulares.

A los 6 años de edad presentó el primer episodio de hemorragia digestiva masiva, que requirió manejo intensivo en Unidad de Cuidados Intensivos Pediátricos. Posteriormente la niña debió ingresar a un programa de ligadura endoscópica, el cual no logró erradicar las várices, presentando múltiples episodios de hemorragia digestiva alta. Con estos antecedentes se decidió realizar una desconexión acigoportal a la edad de 7 años, dada una alteración anatómica que no permitió la realización de un shunt esplenorrenal distal. Durante el procedimiento se realizó una biopsia hepática quirúrgica, para evaluar la evolución, que mostró progresión de la enfermedad

FiguRA 2. Biopsia hepática por aguja. Espacio porta con numerosos conductos biliares de lumen estrecho, separados por abundantes fibras colágenas (Hematoxilina-eosina x 200).

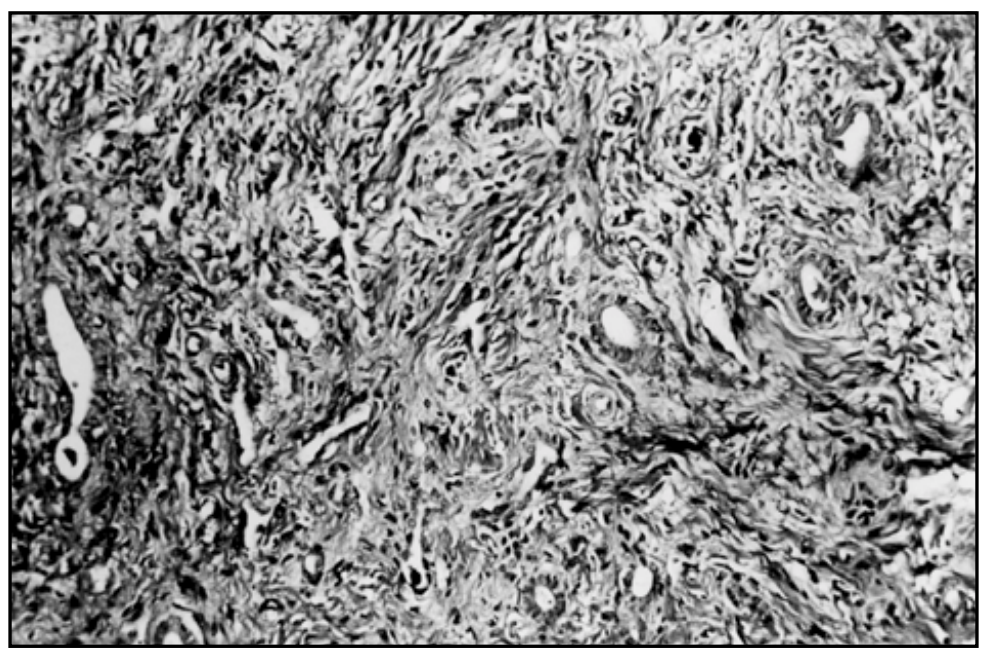




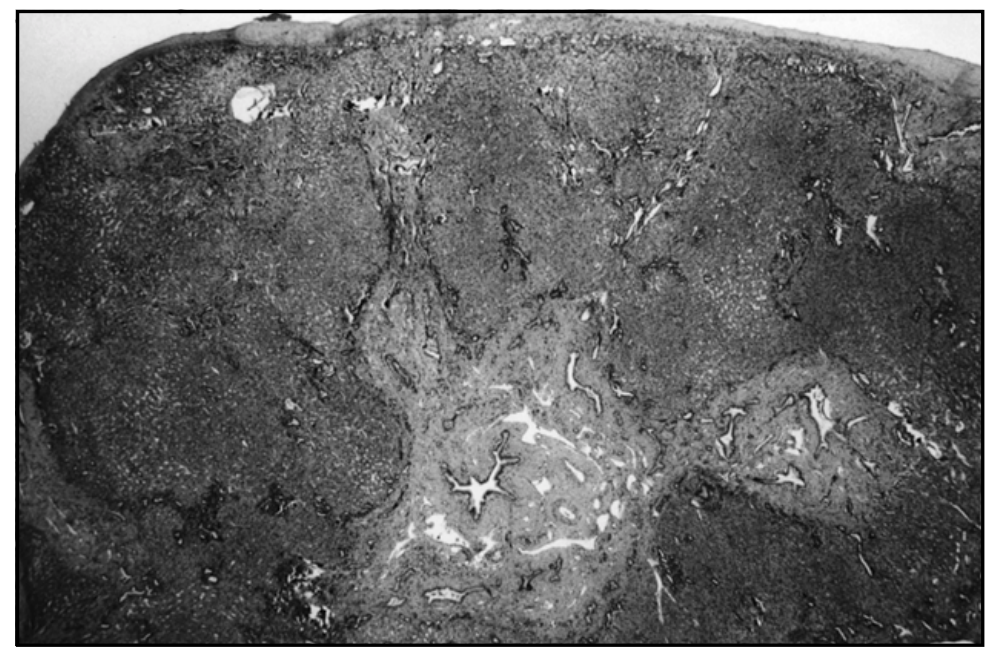

FIgURA 3. Biopsia quirúrgica. Trozo subcapsular de hígado: en el centro y abajo, amplio espacio porta septal ensanchado, fibroso, ramificado, con conductos biliares irregulares. En el resto de tejido se observan pequeños conjuntos de conductos biliares interlobulillares irregulares (Hematoxilina-eosina x 200).

(Figura 3). Posterior a la cirugía, presentó tres episodios de hemorragia digestiva en un período, de 6 meses. Se instaló un TIPS, con lo que se logró controlar las hemorragias y hasta la actualidad no ha vuelto a sangrar. El control ecográfico Doppler del TIPS, a los 18 meses, mostró una estenosis leve, que se resolvió por plastía, vía transyugular, manteniendo función hepática $\mathrm{y}$ renal estables. Desde entonces se controla cada tres meses, evaluando inmunosupresión, función renal y hepática, además con ecografía Doppler según protocolo de TIPS, observando un patrón de crecimiento satisfactorio (peso entre percentil 10-25 y talla en percentil 5, según curvas NCHS). Ha evolucionado sin signos de insuficiencia hepática, pero con crecimiento lento y sostenido de la hepatomegalia, alcanzando $9 \mathrm{~cm}$ bajo el reborde costal a nivel del lóbulo izquierdo, y una esplenomegalia de $4 \mathrm{~cm}$, sin signos de hiperesplenismo, con plaquetas normales y función renal estable. Durante su evolución, la paciente ha presentado bacteremias intermitentes, requiriendo hospitalización y estudio, no pudiendo demostrar puerta de entrada o foco secundario, pero probablemente asociado a translocación bacteriana a nivel intestinal en una paciente inmunosuprimida.

Caso 4: Niño de 14 años portador de ARPKD, diagnosticada en período de recién nacido, que requirió trasplante renal de donante vivo relacionado a los 3 años. En el curso de su evolución, a los 6 años de edad, presentó una hepatomegalia palpable a $4 \mathrm{~cm}$ bajo el reborde costal, principalmente del lóbulo izquierdo, además de una gran esplenomegalia (8 $\mathrm{cm}$ bajo el reborde costal). Como parte del estudio inicial se realizó una biopsia hepática percutánea que confirmó el diagnóstico de FHC y una endoscopia digestiva alta que demostró várices esofágicas grado III y várices en el fondo gástrico. Considerando la esplenomegalia, trombopenia, nivel de actividad física del niño y consideraciones familiares, se optó por un TIPS, como profilaxis de hemorragia, a los 8 años de edad. La última endoscopia, realizada a los 13 años 6 meses de edad, mostró disminución de las várices esofágicas a grado I y ausencia de várices gástricas. Se ha controlado periódicamente cada tres meses, manteniendo un buen desarrollo pondoestatural. No ha presentado sangrado digestivo. El niño se mantiene en excelentes condiciones, que le permiten realizar deporte competitivo. Además, es seguido por protocolo de TIPS con ecografía Doppler dúplex color hepatoportal con transductor electrónico sectorial de 5-2 Mhz, complementado con estudio de flujo mediante Doppler color, Doppler pulsado e imágenes angiográficas, cada tres meses. El shunt se encuentra permeable, sin signos de estenosis, con curvas de velocidad de flujo en rangos normales, los que fluctúan entre 86 y 130 $\mathrm{cm} / \mathrm{s}$. La función hepática se mantiene en límites normales, sin embargo, ha presentado deterioro 
progresivo de su función renal por rechazo crónico del injerto, ingresando a programa de retrasplante. No ha presentado episodio de sangrado variceal.

Caso 5: Niña de 11 años proveniente de La Paz, Bolivia, diagnosticada como portadora de ARPKD, a la edad de 2 años en Estados Unidos. Adicionalmente, la paciente era portadora de un anillo vascular a nivel aórtico, el cual fue intervenido a la edad de 3 años. Posteriormente, se mantuvo en control por insuficiencia renal crónica con manejo médico y sin requerir diálisis. A la edad de 6 años inició episodios aislados de hematemesis que la llevaron a un estudio dirigido, demostrándose la presencia de várices esofágicas grado II, secundarias a una hipertensión portal asociada a enfermedad hepática. Durante dichos episodios de hemorragia digestiva, la paciente fue hospitalizada para manejo médico, requiriendo aporte de volumen, pero sin transfusión de glóbulos rojos o plaquetas. La paciente no fue incorporada a programa de tratamiento endoscópico de las várices. En el curso de los siguientes años, su función renal sufrió un deterioro lento pero progresivo, con regular respuesta a tratamiento médico (eritroproyetina, bicarbonato de sodio, suplementos vitamínicos) y manejo de su hipertensión portal con atenolol. Debido al deterioro de la función renal y a la repercusión importante en la calidad de vida por el cuadro de hipertensión portal, se evaluó en Chile para trasplante renal posterior a solución médico-quirúrgica de la hipertensión portal. La función hepática se presentaba de características normales, en coincidencia con un examen físico que demostraba una hepatomegalia de $3 \mathrm{~cm}$ bajo el reborde costal y un bazo a $7 \mathrm{~cm}$ del mismo. Su recuento de plaquetas se mantenía en niveles de $70.000 \mathrm{x} \mathrm{mm}$. Su última ecografía y angio-resonancia de abdomen demostraba signos evidentes de hipertensión portal, con esplenomegalia y con un aspecto de la vena porta y ramas principales, demostrando una antigua trombosis de vena porta parcialmente recanalizada, con compromiso mayor a nivel de rama portal izquierda, en adición a los hallazgos propios de la enfermedad poliquística renal bilateral. Posterior a una biopsia hepática percutánea, realizada en Santiago, que certificó el diagnóstico al mostrar los hallazgos histológicos característicos y descri- tos en los pacientes previos, se evaluó con equipo de trasplante decidiendo en conjunto con la familia la realización de un shunt quirúrgico de derivación portosistémica a nivel de la vena esplénica (shunt esplenorrenal distal) dado la trombosis de la vena porta que hacían no factible la realización de un shunt del tipo de Rex (reconstrucción venosa portomesentérica, a la rama portal izquierda intrahepática). Posterior a dicha cirugía, la paciente ha evolucionado en buenas condiciones con su tratamiento médico. No ha vuelto a sangrar. El bazo ha disminuido de tamaño y el recuento de plaquetas ha mejorado. Actualmente se encuentra en planificación de trasplante renal con donante vivo.

\section{DisCUSIÓN}

Se presenta una serie de 5 niños con diagnóstico de FHC, tres de ellos en el curso clínico de su enfermedad de base (ARKPD) y dos como sospecha clínica al encontrar una hepatomegalia en un examen físico de rutina. Todos los niños presentaban función hepática normal al momento del diagnóstico. La función renal no estaba comprometida en aquellos dos niños con diagnóstico por hallazgo incidental.

La FHC fue descrita como enfermedad fibroquística del hígado por Grumbach y cols, en 1954, pero la denominación de fibrosis hepática congénita fue introducida por Kerr y cols, en $1961^{4}$. Su prevalencia no ha sido establecida, pero se piensa que es homologable a otras patologías autosómicas recesivas del hígado, como la enfermedad de Wilson (1:100.000 recién nacidos vivos), sin predominancia étnica ni por sexo. La consanguinidad aumenta el riesgo de tener la enfermedad, con padres que son presumiblemente heterocigotos pero fenotípicamente normales ${ }^{3}$.

Cuando se presenta un recién nacido con FHC, las lesiones renales no son frecuentemente evidentes en la infancia o son de menor cuantía; por el contrario, un recién nacido con ARPKD se presentará con lesiones renales clínicamente prominentes. Sin embargo, con el aumento de sobrevida, las lesiones hepáticas en pacientes con ARPKD llegan a ser muy similares a las FHC primarias, lo que ocurrió en los casos clínicos 3, 4 
y 5, sugiriendo que ambas patologías corresponden a una entidad única ${ }^{5-7}$. Los elementos clínicos varían dependiendo de la edad de la primera manifestación; en recién nacidos y niños pequeños predomina la enfermedad renal (ARPKD) y en los niños mayores y adultos predomina la $\mathrm{FHC}^{8,9}$. La enfermedad renal en ARPKD puede variar desde un hallazgo incidental a ser la causa de muerte en un niño mayor. En lactantes con manifestaciones renales de enfermedad poliquística, los riñones están aumentados de tamaño y severamente disfuncionales, pueden desarrollar insuficiencia renal crónica e hipertensión arterial y se presentan con riñones palpables, frecuentemente, desde la primera evaluación ${ }^{10}$. En estos niños, la fibrosis hepática puede ser progresiva pero es de poca importancia; de hecho, en los tres últimos casos que describimos, no se sospechó el diagnóstico de FHC, no obstante el diagnóstico de ARKPD fue realizado en forma precoz. Cabe mencionar que hay comunicaciones de pacientes con cuadro clínico de FHC en quienes la necropsia mostraba una estructura renal normal y tenían antecedentes de función renal normal. Es posible que al aumentar la sobrevida de los niños con ARPKD, como consecuencia del trasplante renal, la FHC tenga relevancia en sus manifestaciones clínicas, permitiendo observar la historia natural de la enfermedad, como sucedió en dos de los pacientes reportados (casos 3 y 4).

La ARPKD tiene una incidencia estimada de 1 en 20.000 nacidos vivos, y es la patología quística renal más frecuente en la edad pediátrica. Se caracteriza por la presencia de dilataciones fusiformes de los ductos colectores del nefrón, que se irradian desde la médula hasta la corteza renal ${ }^{3}$. El gen comprometido es conocido como PKHD1, que se encuentra en el brazo corto del cromosoma 6 y que codifica para una proteína llamada fibrocistina que se postula como receptor que participaría en la diferenciación biliar y de los túbulos colectores ${ }^{11,12}$. La ARKPD puede presentarse como una patología de inicio prenatal, neonatal, infantil o juvenil, estando esta variabilidad relacionada a la expresión variable de mutaciones del mismo gen, como también al efecto de genes modificadores y factores ambientales, más que a mutaciones en diferentes genes ${ }^{13-15}$. Un estudio reciente consigna que la aparición de hipertensión portal ocurrió en 37\% de los niños con ARKPD que sobrevivieron el primer año de vida $^{16}$. A su vez, Khan y cols, en una evaluación de niños trasplantados renales con insuficiencia renal crónica secundaria a ARKPD, reportaron que casi $80 \%$ de estos niños desarrollaron complicaciones relacionadas a la FHC, las cuales ocasionaron $80 \%$ de las muertes de este grupo de estudio $^{17}$.

La enfermedad hepática en FHC se presenta con hipertensión portal, observándose fibrosis hepática 0 anormalidades anatómicas de la vena porta $^{18}$. Entre 30 y 70\% de los casos se presenta como hemorragia digestiva ${ }^{19}$. En niños, la edad de presentación de la hematemesis o melena puede ser tan precoz como desde el primer año de vida, pero la mayor frecuencia se observa entre los $5 \mathrm{y}$ 13 años. La hepatomegalia es característicamente a expensas del lóbulo izquierdo y de consistencia firme, como ocurrió en nuestros 5 pacientes. El hecho de que el crecimiento hepático sea principalmente del lóbulo izquierdo es casi una constante, ya reportada en la literatura ${ }^{20,21}$, sin clara explicación fisiopatológica. La esplenomegalia ocurre en la mayoría de los casos, acompañada de hiperesplenismo con trombocitopenia. La presión de la vena esplénica está aumentada y frecuentemente se forman comunicaciones esplenorrenales o gastrorrenales. Hay que considerar que en nuestros pacientes, estas alteraciones solamente se observaron en los niños con diagnóstico inicial de ARKPD (casos 3, 4 y 5). Adicionalmente, se han descrito anormalidades de la vena porta (duplicación de ramas intrahepáticas) y trombosis de ésta. No obstante lo previamente descrito, la enfermedad puede permanecer silente toda la vida, diagnosticándose por hallazgos en la necropsia. Otros hallazgos constantes en la FHC son las lesiones biliares, característicamente dilatación de ductos intrahepáticos y colangitis, contribuyendo a la morbimortalidad de esta patología 22,23 .

El diagnóstico se debe sospechar en un paciente con hepatomegalia o hipertensión portal con el hígado alargado y firme, con prominencia del lóbulo izquierdo, bazo palpable y ocasionalmente riñones palpables más hipertensión arterial $^{1,2}$. En la mayoría de los niños, los parámetros bioquímicos de síntesis y función hepática son normales, como ocurrió en esta serie, pudiendo 
observarse un leve aumento de transaminasas con bilirrubina normal ${ }^{1,2}$. Cuando se presenta una colangitis crónica se observa aumento del recuento de glóbulos blancos, velocidad de sedimentación, globulinas, nitrógeno ureico y creatinina, asociado a disminución del clearence renal ${ }^{22}$.

En la ecografía Doppler de la vasculatura portal se puede observar signos sugerentes de hipertensión portal, esplenomegalia, intensa ecogenicidad hepática, duplicación de la vasculatura intrahepática, además de evaluar el flujo (velocidad, dirección). En la ecografía renal se puede observar un aumento del tamaño y ecogenicidad de los riñones ${ }^{24}$. Sin embargo, el diagnóstico definitivo de FHC es el estudio histológico, con malformación de la placa ductal ${ }^{18}$.

Los elementos centrales son el tratamiento oportuno y adecuado de la hipertensión portal, trasplante renal en aquellos casos con alteración renal muy importante y trasplante hepático según la evolución y necesidad de cada paciente. Un rol fundamental en el control de la hipertensión portal lo ha asumido la técnica de TIPS $^{25}$. El concepto de un abordaje percutáneo para realizar shunt portosistémicos (en el tratamiento de hemorragia digestiva alta secundaria a várices esofágicas) data de la década 1960-69. Sin embargo, la realización de esta idea se basó posteriormente en el desarrollo de endoprótesis metálicas (stents) que permitieran un adecuado paso de sangre entre el sistema porta y la circulación sistémica representada por las venas suprahepáticas. Estas prótesis permitieron la vía percutánea como mejor forma de instalación. El primer caso exitoso fue reportado en 1988 por Rossle y Richter en la Universidad de Freiburg, Alemania ${ }^{26}$. Esta técnica consiste en establecer una comunicación intrahepática entre el sistema porta y una vena suprahepática, mediante la instalación de un stent metálico, con el objetivo de disminuir la presión sanguínea del sistema porta, logrando así el manejo de las várices esofágicas o la ascitis, según sea el caso.

La experiencia con el uso de TIPS en pediatría es aún escasa, debido principalmente a la ausencia de candidatos que se beneficien de este procedimiento. La mayor parte de los casos reportados son en niños mayores de cinco años, en los cuales se alcanzan tasas de éxito similares a las observadas en adultos, las cuales van entre 75 y $90 \%$ si se considera la correcta instalación del shunt ${ }^{26}$. Las principales indicaciones en niños han sido atresia biliar, que de por sí son malos candidatos, debido a su edad de presentación, su rápida progresión a cirrosis y la alta frecuencia de alteraciones anatómicas en la vena portal en este grupo de pacientes, FHC, síndrome de BuddChiari, y fibrosis quística. Las principales complicaciones constituyen, a corto plazo, el desplazamiento del stent, y a largo plazo la encefalopatía hepática y la oclusión del shunt, menos frecuente en niños la primera, e igual de frecuente que la población adulta la segunda ${ }^{26}$. Esta técnica aparece entonces como una buena manera de manejar la hipertensión portal y sus complicaciones en la población pediátrica, especialmente en aquellos pacientes en espera de trasplante hepático ${ }^{27}$, en especial en la FHC en que la función hepática, es generalmente normal y no se desarrolla una encefalopatía hepática.

En Chile, las primeras descripciones corresponden a 2 niños comunicados por Zacarías y cols, en $1971^{28}$ y en 1984, el de tres casos clínicos en una familia mapuche, descritos por Sierralta y $\operatorname{cols}^{29}$. Sólo recientemente encontramos dos reportes nacionales enfocados en alternativas terapéuticas. González y cols, describen un niño y dos adultos jóvenes con FHC, dos de ellos tratados exitosamente con la operación de Sugiura y uno fallecido por complicaciones infecciosas ${ }^{30}$. Un reciente reporte de Roque y cols, describen un niño con FHC de 12 años con un exitoso trasplante hepático y renal combinado por enfermedad renal terminal ${ }^{31}$.

En esta serie clínica sólo tres pacientes presentaban compromiso renal al momento del diagnóstico y fue en aquellos niños portadores de ARPKD y que, en dos de ellos, requirieron trasplante renal precoz. En ellos, el compromiso hepático apareció en el curso de su enfermedad, como es lo habitual en esta patología. Los niños asintomáticos en los que el diagnóstico se sospechó por el hallazgo clínico de una hepatomegalia persistente, no han presentado compromiso renal evidente hasta la fecha. Todos los niños han evolucionado en buenas condiciones, con un aceptable, pero no óptimo, desarrollo pondoestatural y actividad física adecuada a su edad. Es importante mantener 
un seguimiento periódico para detectar deterioro de la función renal en aquellos niños con diagnóstico reciente. El diagnóstico de la FHC debe sospecharse, no sólo en niños portadores de ARPKD, sino también en niños que presentan una

\section{REFERENCIAS}

1. Zerres K, Rudnick-Schoneborn S, Deget F, Holtkamp U, Brodehl J, Geisert J et al. Autosomal recessive polycystic kidney disease in 115 children: clinical presentation, course and influence of the gender. Acta Paediatr 1996; 85: 437-45.

2. D’Agata I, Jonas M, Pérez-Atayde A, Guay-WooDFORD L. Combined cystic disease of the liver and kidney. Semin Liver Dis 1994; 14: 215-28.

3. Jamil B, McMahon L, Savige J, Wang Y, Walker R. A study of long term morbidity associated with autosomal recessive polycystic kidney disease. Nephrol Dial Transplant 1999; 14: 205-9.

4. Kerr D, Harrison C, SherLock S, Walker R. Congenital hepatic fibrosis. Q J Med 1961; 117: 91-117.

5. KeRR D, Окоnкwo S, CHOA R. Congenital hepatic fibrosis: the long-term prognosis. Gut 1978; 19: 514-20.

6. Lonergan G, Rice R, Sú́rez E. Autosomal recessive polycystic kidney disease: Radiologic-pathologic correlation. Radiographics 2000; 20: 837-55.

7. Alvarez F, Bernard O, Brunele F, Hadchouel M, Leblanc A, Odievre M et al. Congenital hepatic fibrosis in children. J Pediatr 1981; 99: 370-5.

8. Dusol M, Levi J, Giasser K, Schiff E. Congenital hepatic fibrosis with dilation of intrahepatic bile ducts. Gastroenterol 1976; 71: 839-43.

9. MurRay-Lyon I, Ockenden BG, Wiwams R. Congenital hepatic fibrosis, is a single clinical entity? Gastroenterol 1973; 64: 653-6.

10. GANG D, HeRRIN J. Infantile polycystic disease of the liver and kidneys. Cl Nephrology 1986; 25: 2836.

11. Ward C, Hogan M, Rossetti S, Walker D, SNeddon T, WANG X ET AL. The gene mutated in autosomal recessive polycystic kidney disease encodes a large, receptor like protein. Nat Genet 2002; 30: 259-69.

12. Lens $\mathrm{X}$, Onuchic $\mathrm{L}, \mathrm{Wu} G, \mathrm{Gayashi} T$, Daoust $\mathrm{M}$, MochizUKI T ET AL. An integrated genetic and physical map of the autosomal recessive polycys- hepatomegalia persistente, dura y de predominio del lóbulo izquierdo, para iniciar el estudio y manejo adecuado. El pediatra general debe conocer esta enfermedad para derivar en forma oportuna al especialista y no retrasar su manejo.

tic kidney disease region. Genomics 1997; 41: 463-6.

13. Mounif El-Youssef, Yan M, LuUn $X$, Huang $Y$, SteLmach V, GrawFord S. Increased expression of transforming growth factor B1 and thrombospondin-1 in congenital hepatic fibrosis: possible role of the hepatic stellate cell. J Pediatr Gastroenterol Nutr 1999; 28: 386-91.

14. Proesmans W, Van Damme B, Casaer P, Marchal G. Autosomal dominant policystic kidney disease in the neonatal period: Association with a cerebral arteriovenous malformation. Pediatrics 1982; 70 : 971-5.

15. Guay-Woodford L, Gawiani C, Musulman-Mroczeck E, Spear G, Guilot A, Berstein J. Diffuse renal cystic disease in children: morphologic and genetic correlations. Pediatr Nephrol 1998; 12: 173-82.

16. Caine Y, Deckelbaum RJ, Weizman Z, Ljovetsky G, SCHILER M. Congenital hepatic fibrosis unusual presentations. Arch of Disease in Childhood 1984; 59: 1094-6.

17. Khan K, Schwarzenberg S, Sharp H, Matas A, CHAVERS B. Morbidity from congenital hepatic fibrosis after renal transplantation for autosomal recessive polycystic kidney disease. Am J Transplant 2002; 2: 360-5.

18. DeSMET VJ. Congenital diseases of intrahepatic bile ducts: variations on the theme ductal plate malformation». Hepatology 1992; 16: 1069-83.

19. Fiorilo A, Miglorati R, Vajro P, Caldore M, VeCCHIORE R. Congenital hepatic fibrosis with gastrointestinal bleeding in early infancy. Clin Pediatr 1982; 21: 183-5.

20. Chi-Loo L, Kaplowitz N. Diseases of the biliary tree. En: Yamada T, ed. Textbook of Gastroenterology. Philadelphia: Editorial: JB Lippincott Company, 2a edición, 1995; 2212-54.

21. SHerLock S, Dooley J. Cyst and congenital biliary abnormalities. En: Sherlock S, Dooley J, ed. Diseases of the liver and biliary system. London: Blackwell Publishing, 11aㅡ edición, 2002; 583-96. 
22. Sánchez C, González E, Garau J. Trimethoprim sulfamethoxazole treatment of cholangitis complicating congenital hepatic fibrosis. Pediatr Infect Dis 1986; 5: 360-3.

23. LeVy AD, Rohrmann CA JR, MuraKata LA, LoNergan GJ. Caroli's disease: radiologic spectrum with pathologic correlation. Am J Roentgenol 2002; 179: 1053-7.

24. Avni F, Guissard G, Hall M, Janssen F, Demaertelaer V, RYPENSF. Hereditary polycystic kidney diseases in children: changing sonographic patterns through childhood. Pediatr Radiol 2002; 32: 169-74.

25. BOYER TD. Transjugular intrahepatic portosystemic shunt: current status. Gastroenterol 2003; 124: 1700-10.

26. Hackworth C, Leef J, Rosenblum J, Whitington $P$, Miws J, Alonso E. Transjugular intrahepatic portosystemic shunt creation in children: Initial clinical experience. Radiology 1998; 206: 109-14.
27. Heyman M, Laberge J, Somberg K, Rosenthal $P$, Mudge C, Ring E et aL. Transjugular intrahepatic portosystemic shunts (TIPS) in children. J Pediatr 1997; 131: 914-9.

28. Zacarías J, Del Pozo H, Brinck P. Fibrosis hepática congénita (reporte de 2 casos clínicos). Rev Chil Pediatr 1971; 42: 429-32.

29. Sierralta A, Hofmann E, Zelada P, Maraboli O, ArReXT H. Fibrosis hepática congénita. Reporte de 3 casos en una familia araucana. Rev Méd Chile 1984; 112: 157-60.

30. González M, Acuña R, Reyes $H$, Arancibia F, Carvajal $\mathrm{C}$, Donoso S. Fibrosis hepática congénita: una causa inhabitual de hipertensión portal. Rev Méd Chile 1993; 121: 791-4.

31. Roque J, Ríos G, Hepp J, Innocenti F, Humeres R, Palacios JM et aL. Trasplante combinado hepatorrenal: a propósito de un caso. Rev Méd Chile 2003; 131: 1309-12. 